\title{
Leaching models for multiple immersed materials and for granular materials flushed in a column
}

\author{
H.J.H. Brouwers \\ Department of Civil Engineering \& Management, University of Twente, P.O. Box 217, 7500 AE Enschede, \\ Netherlands
}

Received 14 May 1996; revised 5 October 1996; accepted 5 October 1996

\begin{abstract}
The present paper addresses the leaching of hazardous contaminants from immersed and replenished materials and from granular materials flushed in a column. First, the leaching of an immersed material in contact with a limited volume of leachant is studied. The mass transfer from material to leachant is assumed to be inversely proportional to $\sqrt{ } t$ (i.e. following the semiinfinite medium diffusion model). The leaching model accounts for the concentration of the contaminant in the leachant, the (deviation from) equilibrium partition of the contaminant between material and leachant, and leachant replenishment. The governing equations are solved in closed form, yielding the contaminant concentration in leachant and monolith versus the elapsed time. For special cases this solution corresponds to the leaching expressions obtained by Godbee and Joy (1974). Subsequently, the unsteady leaching process from a granular material packed in a column, flushed by a leachant, is modeled. Here, the mass transfer from material to leachant is also assumed as inversely proportional to $\sqrt{ } t$. The model leads to a moving boundary problem, the governing partial differential equations are transformed and solved using asymptotic techniques. Approximate expressions are obtained for the contaminant concentration of the material and in the leachant. Of special practical interest is the leachant concentration at the exit of the column as here the leachant can be collected in flasks and analyzed. Finally, the models are generalized to systems where the mass transfer is an arbitrary power function of time. The resulting equations can for instance be used for determining an effective diffusion coefficient and/or comparing immobilization yields. C) 1997 Elsevier Science B.V.
\end{abstract}

Keywords: Leaching model; Immersed material; Granular material; Stabilization/solidification

\section{Introduction}

Immobilization processes are used to render hazardous wastes less harmful to the environment. In the U.S., these processes are generally referred to as chemical stabiliza- 
tion/solidification (or S/S). Although waste reduction and waste recycle are preferable, they are not always possible. A first application of immobilization concerned the treatment of radioactive wastes. Recently, immobilization is carried out to treat waste streams of various industries in order to render these streams suitable either for secure landfill or for application in the building and construction industry. Though the leaching of the hazardous contaminants from the waste may be greatly reduced by immobilization, the contaminant species are still available for slow leaching into the environment.

To assess the long-term behavior of immobilisates, short-term tests have been developed. In the Netherlands, a standard set of leaching tests are in use. For monoliths the standard [1] is developed, whereas for granular materials standard [2] is used. These tests have been developed to assess the environmental impact of building materials, especially if hazardous waste is incorporated. [1] comprises a serial batch leaching test whereby the monolith is immersed in a bath which is replenished at the start of each cycle. This test is very similar to the U.S. leaching test developed for radioactive waste, [3]. [2] concerns the flushing of granular materials which are packed and flushed in a vertical column. The leachant that leaves the column is then collected in flasks. In other countries similar tests are in use. An overview and experimental comparison is offered by [4].

To interpret the experimental results mechanistic models are needed from which an effective diffusion coefficient can be derived. [5] were the first to derive a model for the leaching of monoliths. This model was based on the solution of the second order diffusion model of a semiinfinite medium. Their expressions are widely used to evaluate the leaching tests and adopted by regulatory agencies. [6] obtained original results for leaching, including matrix dissolution and finite leachant volume, using a first order (pseudo-steady state) diffusion model. [7] described the leaching from granular materials. Their model was based on non-equilibrium between leachant and solid material, but the mass transfer coefficient was assumed to be constant. Recently, [8] used a shrinking core model in order to describe the leaching from an immersed dissoluting monolithic material.

The presented models account for the diffusion of contaminant in the material and the mass transfer between material and leachant. To this end, the common chemical engineering assumption is employed that mass transfer between material and leachant is proportional to the difference between equilibrium and actual concentrations. Equilibrium between both phases is described with the help of a partition coefficient. The mass transfer is governed by the slow diffusion in the material. This diffusion is described by using the second order diffusion model of a semiinfinite material. For the monolith leaching the present approach yields a solution in closed form for the contaminant concentrations in monolith and leachant as a function of time. Furthermore, it is demonstrated that for a special case the expressions can be simplified to the standard equations of [5]. The granular materials leaching model yields original analytic expressions for the concentrations in monolith and leachant. These explicit results reveal the principle phenomena that govern this process and enable the derivation of an effective diffusion coefficient of the granular material.

The mass transfer coefficient used is taken from the second order diffusion model of a semiinfinite material, resulting in a mass transfer that is inversely proportional to $\sqrt{ } t$. 
The results of the present analysis are however also directly applicable if other leaching mechanisms are considered whereby mass transfer is inversely proportional to $\sqrt{ } t$. Examples are the pseudo steady state diffusion models of [6] and [8], which result in expressions for the mass transfer which can be inserted directly in the present models. In a special section the models are generalized to situations where mass transfer is proportional to $t^{\mu-1}$.

\section{Leaching of an immersed material}

In this section a model is derived for the leaching behavior of a material containing leachates. These materials are tested to judge the leaching of inorganic micropollutants under fields conditions. According to Dutch Standard [1] monolithic materials are subjected to a serial batch leaching test. During this test the sample with mass $S$ is immersed in a nonagitated bath containing acidified water $(\mathrm{pH}=4)$ of volume $L$ as leachant (Fig. 1). The first leachant is replenished by fresh leachant after $6 \mathrm{~h}$. Subsequently, the leachant is replenished $24 \mathrm{~h}$ after the start, and subsequently 2.25 days, 4 days, 9 days, 16 days, 36 days and 64 days after the start of the test. In total, the test thus comprises 8 leaching cycles (Table 1). After each cycle, the concentration of the various hazardous contaminants in the leachant is determined.

For the leachant, during leaching cycle $i$, a differential mass balance of contaminant gives:

$$
L_{i} \frac{\mathrm{d} c_{1}}{\mathrm{~d} t}=A \dot{m} .
$$

In this equation the first term accounts for the accumulation of contaminant in the leachant, $A$ is the interfacial area between material and leachant, and $\dot{m}$ is the mass flux between both phases. The leachant is assumed to be perfectly mixed, i.e. the bath of homogeneous composition. Neglecting the mass transfer resistance at the leachant side, the mass transfer can be expressed as

$$
m=k_{\mathrm{s}} \rho_{\mathrm{s}}\left(C_{\mathrm{s}}-K_{\mathrm{p}} c_{1}\right) \text {. }
$$

In this equation $K_{\mathrm{p}}$ represents the partition coefficient of the contaminant in leachant and material. One can readily see that no contaminant is transported in case equilibrium prevails, that is when $C_{\mathrm{s}}=K_{\mathrm{p}} c_{1}$. For small $4 \mathbb{D}_{\mathrm{s}} t / b^{2}$, where $b$ is a characteristic length

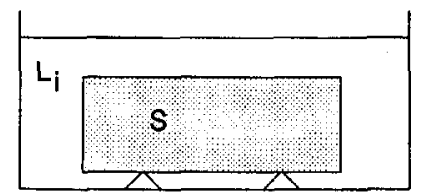

Fig. 1. Schematic representation of a monolith immersed in a bath. 
Table 1

Start and end times pertaining to the leaching of monolithic materials according to NEN 7345 ([1])

\begin{tabular}{llll}
\hline Leaching cycle $i$ & Start of $i: t_{i-1}$ (days) & End of $i: t_{i}$ (days) & $\sqrt{ } t_{i}-\sqrt{ } t_{i-1}$ ( days) \\
\hline 1 & 0 & 0.25 & 0.5 \\
2 & 0.25 & 1 & 0.5 \\
3 & 1 & 2.25 & 0.5 \\
4 & 2.25 & 4 & 0.5 \\
5 & 4 & 9 & 1 \\
6 & 9 & 16 & 1 \\
7 & 16 & 36 & 2 \\
8 & 36 & 64 & 2 \\
\hline
\end{tabular}

of the monolith, the mass transfer coefficient follows from the semiinfinite medium model ([9]) as:

$$
k_{\mathrm{s}}=\left(\frac{\mathbb{D}_{\mathrm{s}}}{\pi t}\right)^{1 / 2} \text {. }
$$

In chemical engineering literature the theory underlying this expression is referred to as the penetration theory ([10]). The length $b$ may be the average rib length of a rectangular block or the diameter of a spherical object. A mass balance for the monolithic material yields:

$$
S \frac{\mathrm{d} C_{\mathrm{s}}}{\mathrm{d} t}=-A \dot{m} .
$$

In this equation the first term accounts for the decay of contaminant in the material. The initial conditions at the start of leaching cycle $i$ (from $t_{i-1}$ to $t_{i}$ ), $i=1, \ldots, 8$ reads:

$$
\begin{aligned}
& c_{\mathrm{l}}\left(t_{i-1}\right)=0, \\
& C_{\mathrm{s}}\left(t_{i-1}\right)=C_{\mathrm{s}(i-1)} .
\end{aligned}
$$

An overall mass balance of contaminant in material and leachant yields:

$$
C_{\mathrm{s}}(t)=C_{\mathrm{s}(i-1)}-\frac{L_{i}}{S} c_{1}(t)
$$

This equation could also have been obtained by combining Eqs. (1) and (4) to eliminate $A \dot{m}$, integrating with respect to $t$, and applying Eqs. (5) and (6).

Eqs. (2), (3) and (7) are substituted into Eq. (1) yielding:

$$
L_{i} \frac{\mathrm{d} c_{1}}{\mathrm{~d} t}=\rho_{\mathrm{s}} A\left(\frac{\mathbb{D}_{\mathrm{s}}}{\pi t}\right)^{1 / 2}\left(C_{\mathrm{s}(i-1)}-\left(K_{\mathrm{p}}+\frac{L_{i}}{S}\right) c_{1}\right) .
$$

Separation of the variables $c_{1}$ and $t$, integration and application of Eq. (5) yields:

$$
c_{1}(t)=\frac{L_{i}}{S} \frac{C_{\mathrm{s}(i-1)}}{\left(1+\frac{S K_{\mathrm{p}}}{L_{i}}\right)}\left(1-\exp \left(-\frac{\rho_{\mathrm{s}} A b}{S}\left(1+\frac{S K_{\mathrm{p}}}{L_{i}}\right) \epsilon\right)\right),
$$


with

$$
\epsilon=\left(\frac{4 \mathbb{D}_{\mathrm{s}}}{\pi b^{2}}\right)^{1 / 2}\left(\sqrt{ } t-\sqrt{ } t_{i}\right)
$$

Eq. (9) represents the contaminant concentration in the leachant for $t_{i-1} \leq t \leq t_{i}$. The magnitude of $A b$ is about $V / 6$. As $S / V=\rho_{\mathrm{s}}$, it can be concluded that the factor $\rho_{\mathrm{s}} A b / S$ is smaller than unity. Furthermore, if it is assumed that $1+S K_{\mathrm{p}} / L_{\mathrm{i}}$ is of order unity, and by virtue of $\epsilon$ being small (this assumption underlies the semiinfinite material diffusion model used here), the exponential power in Eq. (9) is expanded as a Taylor series, yielding:

$$
c_{1}(t)=\frac{2 \rho_{\mathrm{s}} A C_{\mathrm{s}(i-1)}}{L_{i}}\left(\frac{\mathbb{D}_{\mathrm{s}}}{\pi}\right)^{1 / 2}\left(\sqrt{ } t-\sqrt{ } t_{i-1}\right)+O\left(\epsilon^{2}\right) .
$$

The contaminant mass fraction in the material for $t_{i-1} \leq t \leq t_{i}$ follows from Eqs. (7) and (11) as:

$$
C_{\mathrm{s}}(t)=C_{\mathrm{s}(i-1)}\left(1-\frac{2 \rho_{\mathrm{s}} A}{S}\left(\frac{D_{\mathrm{s}}}{\pi}\right)^{1 / 2}\left(\sqrt{ } t-\sqrt{ } t_{i-1}\right)\right)+O\left(\epsilon^{2}\right) .
$$

At the end of leaching cycle $i, t=t_{i}$, the first order approximation of the contaminant mass fraction $C_{\mathrm{s}}\left(t_{i}\right)\left(=C_{\mathrm{s} i}\right)$ reads:

$$
C_{\mathrm{s} i}=C_{\mathrm{s}(i-1)}\left(1-\frac{2 \rho_{\mathrm{s}} A}{S}\left(\frac{\mathbb{D}_{\mathrm{s}}}{\pi}\right)^{1 / 2}\left(\sqrt{ } t_{i}-\sqrt{ } t_{i-1}\right)\right),
$$

which corresponds to the mass fraction at the start of leaching cycle $i+1$. In order to relate $c_{1}(t)$ and $C_{\mathrm{s}}(t)$ to the mass fraction in the material at the start of the test, i.e. at the start of the first leaching cycle, to which applies $i=1, t=t_{0}=0$ and $C_{\mathrm{s}}=C_{\mathrm{s} 0}$, Eqs. (12) and (13) are combined:

$$
C_{\mathrm{s}}(t)=C_{\mathrm{s} 0}-\frac{2 \rho_{\mathrm{s}} A C_{s 0}}{S}\left(\frac{\mathbb{D}_{\mathrm{s}}}{\pi}\right)^{1 / 2} \sqrt{ } t+O\left(\epsilon^{2}\right),
$$

which is valid throughout the entire test period $\left(0 \leq t \leq t_{8}\right)$. Note that $\left(C_{\mathrm{s} 0}-C_{\mathrm{s}}(t)\right) S$ represents the mass of contaminant that is leached out and that $\left(C_{\mathrm{s} 0}-C_{\mathrm{s}}(t)\right) / C_{\mathrm{s} 0}$ constitutes the ratio of removed contaminant and the initial contaminant present in the material.

As a first order approximation for small $\epsilon$, the concentration in the leachant during leaching cycle $i$ is obtained by using Eqs. (11) and (13):

$$
c_{1}(t)=\frac{2 \rho_{\mathrm{s}} A C_{\mathrm{s} 0}}{L_{i}}\left(\frac{\mathbb{D}_{\mathrm{s}}}{\pi}\right)^{1 / 2}\left(\sqrt{ } t-\sqrt{ } t_{i-1}\right) .
$$

At the end of this cycle, the concentration is as follows:

$$
c_{1}\left(t_{i}\right)=\frac{2 \rho_{\mathrm{s}} A C_{\mathrm{s} 0}}{L_{i}}\left(\frac{\mathbb{D}_{\mathrm{s}}}{\pi}\right)^{1 / 2}\left(\sqrt{ } t_{i}-\sqrt{ } t_{i-1}\right)
$$


which can be measured by analysing the eluate. Note that Eqs. (11) and (15) are equivalent, but that in the former $C_{\mathrm{s}(i-1)}$ appears and in the latter $C_{\mathrm{s} 0}$. This follows from the fact that for $c_{1}$, unlike $C_{\mathrm{s}}$, a zero-order solution for small $4 \mathbb{D}_{\mathrm{s}} t / b^{2}$ is absent.

Furthermore, $c_{1}\left(t_{i}\right) L_{i}$ represents the total mass of contaminant that has entered the leachant during leaching cycle $i$. The total mass of leached contaminant after $n$ leaching cycles reads:

$$
M_{\mathrm{l}}\left(t_{n}\right)=\sum_{i=1}^{n} L_{i} c_{1}\left(t_{i}\right)=2 \rho_{\mathrm{s}} A C_{\mathrm{s} 0}\left(\frac{\mathbb{D}_{\mathrm{s}}}{\pi}\right)^{1 / 2} \sqrt{ } t_{n},
$$

where Eq. (16) has been substituted. This result was first obtained by [5] and is used in [3]. In both references $\rho_{\mathrm{s}} C_{\mathrm{s} 0}$ is replaced by the equivalent quotient $M_{\mathrm{s} 0} / V$, whereby $M_{\mathrm{s} 0}$ is the contaminant mass initially present in the monolith.

The value of $M\left(t_{n}\right)$ does not depend on the volumes of leachant used during each cycle, but mainly on the exposed surface A. [1], however, prescribes the use of an equal volume of leachant $L$ for each cycle, whereby $4 V \leq L \leq 6 V$. Following [1] $\mathscr{D}_{\mathrm{s}}$ (in $\mathrm{m}^{2} / \mathrm{s}$ ) is then computed with the aid of Eq. (16) for each leaching cycle and $-{ }^{10} \log \mathbb{D}_{\mathrm{s}}$ determined. Subsequently, the arithmetic mean of all computed $-{ }^{10} \log \mathbb{D}_{\mathrm{s}}$ is than determined, which is used to assess the long-term leaching behavior.

Finally, by combining Eqs. (2), (2), (14) and (15) a first order approximation for the mass flux is now obtained:

$$
\dot{m}=\rho_{\mathrm{s}}\left(\frac{\mathbb{D}_{\mathrm{s}}}{\pi t}\right)^{1 / 2} C_{\mathrm{s} 0} .
$$

This expression holds throughout the entire test period.

In this section the leaching from one monolith has been considered. The present analysis also holds if several identical materials are placed in the bath, for example grains. An effective diffusion coefficient can still be derived using Eq. (17), in which for A the total exposed surface of all material parts should be taken.

\section{Leaching of packed granular materials}

Dutch Standard [2] prescribes the measurement of leaching behavior of granular materials containing inorganic contaminants. To this end, a vertical cylindrical column is filled with the granular material with total dry mass $m_{0}$ and of which at least $95 \%$ ( $w / w$ ) of the grains are smaller than $4 \mathrm{~mm}$. The column is mounted with screens to prevent the passage of grains. The inner diameter of the column $d$ amounts to $50 \pm 5$ mm and the height $l$ is at least four times the inner diameter (Fig. 2). At $t=0$ the column is filled from bottom to top with acidified water. After saturation of the column the flow is continued until $0.110^{-3} \mathrm{~m}^{3} / \mathrm{kg} m_{0}$ has left the column at the top. The leachant that has left the column, denoted as $k_{1}$, is collected in the first flask. The process is continued until again $0.110^{-3} \mathrm{~m}^{3} / \mathrm{kg} m_{0}$ of leachant is flushed, this fraction is collected as $k_{2}$. In total, 7 flasks are filled, the flushing quantities being summarized in Table 2. 


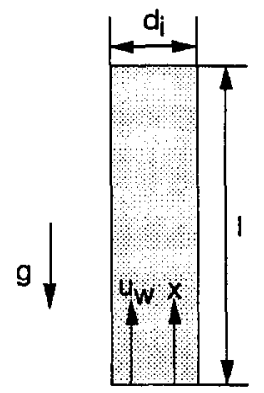

Fig. 2. Schematic representation of a granular material packed and flushed in a column.

For the leachant, a differential mass balance of contaminant gives:

$$
\phi \frac{\partial c_{1}}{\partial t}+u_{1} \frac{\partial c_{1}}{\partial x}=\dot{m} a=a k_{\mathrm{s}} \rho_{\mathrm{s}}\left(C_{\mathrm{s}}-K_{\mathrm{p}} c_{1}\right) \text {. }
$$

In this equation the first term accounts for the accumulation of contaminant in the leachant and the second term the convective transport by the leachant, $\phi$ is the porosity and $m$ is the mass flux, taken from Eq. (2). The mass transfer coefficient $k_{s}$, which depends on the position in the column, will be specified further. In Eq. (19), $a$ represents the interface between material and leachant in the column per volume of column and follows from:

$$
a=\frac{s(1-\phi)}{d_{\mathrm{p}}},
$$

with $d_{\mathrm{p}}$ as mean grain size and $s$ as shape factor ([11]). The value of $s$ ranges from 6 for spherical to 7.7 for angular grains. The mean grain size $d_{\mathrm{p}}$ follows from sieving the grains with a set of rated sieves as:

$$
d_{\mathrm{p}}=\left(\sum \frac{w_{i}}{z_{i}}\right)^{-1},
$$

where $w_{i}$ represents the mass fraction of grains between two $i$ sieves of diameter $z_{1}$ and $z_{2}$, and $z_{i}$ the geometric mean of the sieves, that is $\sqrt{z_{1} z_{1}}$.

Table 2

Volumes of leachant collected in bottles after column is saturated with leachant $\left(L_{j}\right)$ and cumulative volumes according to NEN 7343 ([1]) and pertaining filling times $\left(q=0.02510^{-3} \mathrm{~m}^{3} / \mathrm{kg} m_{0}\right)$

\begin{tabular}{llccc}
\hline Flask $j$ & $\begin{array}{l}\mathrm{L}_{j} / \mathrm{m}_{0} \\
\left(10^{-3} \mathrm{~m}^{3} / \mathrm{kg}\right)\end{array}$ & $\begin{array}{l}\left(\sum \mathrm{L}_{j}\right) / \mathrm{m}_{0} \\
\left(10^{-3} \mathrm{~m}^{3} / \mathrm{kg}\right)\end{array}$ & $\begin{array}{l}\text { Filling time } \\
t_{j}-t_{j-1}(\mathrm{~h})\end{array}$ & $\begin{array}{l}\text { Time } \\
t_{j}-\tau(\mathrm{h})\end{array}$ \\
\hline 1 & 0.1 & 0.1 & 4 & 4 \\
2 & 0.1 & 0.2 & 8 & 12 \\
3 & 0.3 & 0.5 & 20 & 32 \\
4 & 0.5 & 1.0 & 40 & 72 \\
5 & 1.0 & 2.0 & 80 & 152 \\
6 & 3.0 & 5.0 & 200 & 352 \\
7 & 5.0 & 10.0 & 400 & 752 \\
\hline
\end{tabular}


A mass balance for the granular material yields:

$$
(1-\phi) \rho_{\mathrm{s}} \frac{\partial C_{\mathrm{s}}}{\partial t}=-\dot{m} a=-a k_{\mathrm{s}} \rho_{\mathrm{s}}\left(C_{\mathrm{s}}-K_{\mathrm{p}} c_{1}\right) \text {. }
$$

During the filling of the column Eqs. (19) and (22) stay in the region $(0 \leq x \leq \chi \mathrm{t})$ ), where $\chi(t)$ represents the moving front of the leachant saturated part of the column. From an overall mass balance the front position follows as:

$$
\chi(t)=\frac{u_{1} t}{\phi}
$$

It is also possible to determine the time $T$ needed for the leachant front to attain a given position $x$ in the column:

$$
r(x)=\frac{x \phi}{u_{1}}
$$

With this equation one can derive the time required to fill the column, i.e. when $x=l$ :

$$
\tau=\frac{l \phi}{u_{1}} .
$$

It is interesting to note that the ratio $t / \tau$ governs the number of flushed pore volumes.

The boundary conditions pertaining to Eqs. (19) and (22) read:

$$
\begin{aligned}
& c_{1}(t, x=0)=0, \\
& C_{\mathrm{s}}(t, x=\chi(t))=C_{\mathrm{s} 0},
\end{aligned}
$$

respectively. Eq. (26) accounts for the clean leachant at the entrance of the column, and Eq. (27) reflects the initial contaminant concentration in the material.

Leaching of the material in the column starts as soon as the leachant front has attained that particular position. This implies that the leaching time depends on the position in the column. This time follows from the elapsed time since the test was started minus the time needed for the leachant front to attain the position in question. Hence, the mass transfer coefficient reads:

$$
k_{\mathrm{s}}=\left(\frac{\mathbb{D}_{\mathrm{s}}}{\pi(t-\Upsilon(x))}\right)^{1 / 2}
$$

Note that for any position in the column $t-\Upsilon(x)$ is the time measured from the instant that the entering leachant front has reached the position in question. The employed mass transfer coefficient is based on the semiinfinite material model, which is applicable if $4 \mathbb{D}_{\mathrm{s}} t / d_{\mathrm{p}}^{2}$ is small ([9]). In practice this condition is more difficult to fulfil for granular materials than for monoliths as $d_{\mathrm{p}}$ is much smaller than $b$.

Eqs. (19), (22), (26) and (27) represent a moving boundary problem $(t \geq 0,0 \leq x \leq$ $\chi(t)$ ). These equations are transformed by introducing:

$$
c_{\mathrm{s}}=\frac{C_{\mathrm{s}}}{K_{\mathrm{p}}}
$$




$$
\begin{aligned}
& X=\frac{x}{l}, \\
& T=\frac{\chi(t)}{l}-\frac{x}{l}=\frac{t}{\tau}-\frac{x}{l} .
\end{aligned}
$$

$T=0$ corresponds to the position of the boundary between leachant saturated part and dry part of the column, which is described by $\chi(t)$ (see Eq. (23)). Eqs. (19), (22), (26) - (28) in terms of the transformed coordinates now are:

$$
\begin{aligned}
& \frac{\partial c_{1}}{\partial X}=\epsilon_{1} \frac{1}{\sqrt{T}}\left(c_{\mathrm{s}}-c_{1}\right), \\
& \frac{\partial c_{\mathrm{s}}}{\partial T}=-\epsilon_{2} \frac{1}{\sqrt{T}}\left(c_{\mathrm{s}}-c_{1}\right), \\
& c_{1}(T, X=0)=0, \\
& c_{\mathrm{s}}(T=0, X)=\frac{C_{\mathrm{s} 0}}{K_{\mathrm{p}}},
\end{aligned}
$$

with

$$
\begin{aligned}
& \epsilon_{1}=\rho_{\mathrm{s}} K_{\mathrm{p}} a\left(\frac{\mathbb{D}_{\mathrm{s}} l}{\phi u_{1} \pi}\right)^{1 / 2}, \\
& \epsilon_{2}=\frac{\phi}{1-\phi} a\left(\frac{\mathbb{D}_{\mathrm{s}} l}{\phi u_{1} \pi}\right)^{1 / 2} .
\end{aligned}
$$

Eqs. (32)-(35) are similar to the equations encountered in crossflow heat transfer ([12]). The latter equations are however somewhat less complex as they do not contain the $1 / \sqrt{T}$ factor on the right-hand sides. In the past analytical solutions have been derived of this simpler system, starting with [13]. For Eqs. (32)-(35) on the other hand, an analytic solution is difficult or even impossible to obtain. Rather than solving these equations numerically for all $\epsilon_{1}$ and $\epsilon_{2}$, an approximate solution is derived using asymptotic techniques ([14]).

Assuming that $\epsilon_{1}$ and $\epsilon_{2}$ are small, this assumption is verified a posteriori, the following perturbation expansions are substituted into Eqs. (32)-(35):

$$
\begin{aligned}
& c_{1}=c_{1}^{00}+\epsilon_{1} c_{1}^{10}+\epsilon_{2} c_{1}^{01}+O\left(\epsilon_{1} \epsilon_{2}\right), \\
& c_{\mathrm{s}}=c_{\mathrm{s}}^{00}+\epsilon_{1} c_{\mathrm{s}}^{10}+\epsilon_{2} c_{\mathrm{s}}^{01}+O\left(\epsilon_{1} \epsilon_{2}\right),
\end{aligned}
$$

equating the coefficients of equal power of $\epsilon_{1}$ and $\epsilon_{2}$, solving the equations of zeroand first order, and application of boundary conditions (34) and (35) yields:

$$
\begin{aligned}
& c_{\mathrm{l}}^{00}=0, \\
& c_{\mathrm{s}}^{00}=\frac{C_{\mathrm{s} 0}}{K_{\mathrm{p}}},
\end{aligned}
$$




$$
\begin{aligned}
& c_{1}^{10}=\frac{C_{\mathrm{s} 0} X}{K_{\mathrm{p}} / T}, \\
& c_{\mathrm{s}}^{10}=0, \\
& c_{1}^{01}=0, \\
& c_{\mathrm{s}}^{01}=\frac{-2 C_{\mathrm{s} 0} / T}{K_{\mathrm{p}}} .
\end{aligned}
$$

Combining Eqs. (36)-(38), (40), (42) and (44) yields as a first order approximation of the contaminant concentration:

$$
c_{1}(T, X)=\rho_{\mathrm{s}} a C_{\mathrm{s} 0}\left(\frac{\mathbb{D}_{\mathrm{s}} l}{\phi u_{1} \pi}\right)^{1 / 2} \frac{X}{\sqrt{T}},
$$

and by combining Eqs. (29), (36), (37), (39), (41), (43) and (45) a first order approximation for the contaminant mass fraction is obtained:

$$
C_{\mathrm{s}}(T, X)=C_{\mathrm{s} 0}-2 a C_{\mathrm{s} 0} \frac{\phi}{1-\phi}\left(\frac{\mathbb{D}_{\mathrm{s}} l}{\phi u_{1} \pi}\right)^{1 / 2} \sqrt{T} .
$$

In terms of the variables $t$ and $x$, the contaminant concentration in the leachant follows from Eqs. (24), (25), (30), (31) and (46) as:

$$
c_{1}(t \geq 0,0 \leq x \leq \chi(t))=\frac{\rho_{\mathrm{s}} a C_{\mathrm{s} 0} x}{u_{1}}\left(\frac{\mathbb{D}_{\mathrm{s}}}{\pi}\right)^{1 / 2} \frac{1}{\sqrt{ }(t-\Upsilon(x))} .
$$

At the exit of the column, i.e. $X=1$ or $x=1$, from the moment the column is completely filled and the first leachant leaves the column, i.e. $T \geq 0$ or $t \geq \tau$, the exit concentration now follows from Eqs. (24), (25) and (48) as:

$$
c_{1}(t \geq \tau, x=l)=\frac{\rho_{\mathrm{s}} a C_{\mathrm{s} 0} l}{u_{1}}\left(\frac{\mathbb{D}_{\mathrm{s}}}{\pi}\right)^{1 / 2} \frac{1}{\sqrt{ }(t-\tau)} .
$$

The mixed mean concentration in a bottle $k_{j}$ which is filled $t_{j-1}$ and $t_{j}$ is determined via:

$$
\bar{c}_{1}\left(t_{j}\right)=\frac{1}{t_{j}-t_{j-1}} \int_{t_{j-1}}^{t_{j}} c_{1} \mathrm{~d} t .
$$

Substituting Eq. (49) into Eq. (50) and integrating yields:

$$
\bar{c}_{1}\left(t_{j}\right)=\frac{2 \rho_{\mathrm{s}} a C_{\mathrm{s} 0} l}{u_{1}}\left(\frac{\mathbb{D}_{\mathrm{s}}}{\pi}\right)^{1 / 2}\left(\frac{\sqrt{ }\left(t_{j}-\tau\right)-\sqrt{ }\left(t_{j-1}-\tau\right)}{t_{j}-t_{j-1}}\right) .
$$


In order to express $\bar{c}_{1}\left(t_{j}\right)$ in the amount of collected leachant, the following equations are substituted:

$$
\begin{aligned}
& q=u_{1} \frac{\pi}{4} d_{i}^{2}, \\
& q=\frac{L_{j}}{t_{j}-t_{j-1}},
\end{aligned}
$$

yielding:

$$
\bar{c}_{1}\left(t_{j}\right)=\frac{2 \rho_{\mathrm{s}} a C_{\mathrm{s} 0} l \frac{\pi}{4} d_{\mathrm{i}}^{2}}{L_{j}}\left(\frac{\mathbb{D}_{\mathrm{s}}}{\pi}\right)^{1 / 2}\left(\sqrt{ }\left(t_{j}-\tau\right)-\sqrt{ }\left(t_{j-1}-\tau\right)\right) .
$$

The total amount of leached contaminant at $t_{n}$ follows from:

$$
M_{1}\left(t_{n}\right)=\sum_{j=1}^{n} L_{j} \bar{c}_{1}\left(t_{j}\right)=2 \rho_{\mathrm{s}} C_{\mathrm{s} 0} a l \frac{\pi}{4} d_{\mathrm{i}}^{2}\left(\frac{\mathbb{D}_{\mathrm{s}}}{\pi}\right)^{1 / 2} \sqrt{ }\left(t_{n}-\tau\right) .
$$

This equation is very similar to Eq. (17). The factor $l(\pi / 4) d_{\mathrm{i}}^{2}$ represents the volume of the column, and hence, the factor $a l(\pi / 4) d_{\mathrm{i}}^{2}$ the total exposed surface of the material to the leachant in the column. This surface corresponds to the total surface $A$ between monolith and leachant, appearing in Eq. (17). Accordingly, the amount of leached contaminant for monolithic and granular materials is governed by similar relations, which describe the total amount of leached contaminant to be parabolic in time.

$M_{1}\left(t_{n}\right)$ mainly depends on the elapsed time, which is a consequence of the applied model. Eq. (55) reveals that the amount of leached contaminant does not depend on the quantity of flushed leachant used, nor on the flush rate. The mass of leached contaminant mainly depends on the specific surface interfacial area and the volume of the column. The specific area, see Eq. (20), is minimized if spherical grains (minimal $s$ ) and large grains (large $d_{\mathrm{p}}$ ), i.e. $95 \%$ near $4 \mathrm{~mm}$, are used. Moreover, if also grains of uniform sizes are used a large porosity can be created, which will also yield a favorable leaching result. NEN 7343 ([2]), however, does not account for these effects. Furthermore, the volume of the column can be reduced by using the prescribed minimum length, namely $l=4 d_{\mathrm{i}}$, and the minimum diameter, namely $d_{\mathrm{i}}=50 \mathrm{~mm}$.

Now it is interesting to investigate how the collected quantities of leachant, listed in Table 2, are related to the time to fill the column, and the times needed to fill flasks 1 to 7. Following [2] the volume flow rate is related to the material mass via:

$$
q=\alpha m_{0},
$$

with as allowed maximum $\alpha=0.02510^{-3} \mathrm{~m}^{3} / \mathrm{kg} \mathrm{h}$. Using Eq. (56), $\alpha=0.02510^{-3}$ $\mathrm{m}^{3} / \mathrm{kg} \mathrm{h}$ and the tabulated values of $L_{j}$, values of $t_{j}-t_{j-1}$ are computed and included in Table $2\left(t_{0}\right.$ is start time of filling of flask 1 , that is $t=t_{0}=\tau$ ). As both $L_{j}$ and $q$ depend linearly on $m_{0}, t_{j}-t_{j-1}$ depends on $\alpha$ only.

To determine $\tau$, use is made of the definition of $\phi$ :

$$
m_{0}=(1-\phi) l \frac{\pi}{4} d_{\mathrm{i}}^{2} \rho_{s} \text {. }
$$


Combining Eqs. (25), (52), (56) and (57) yields as minimum saturation time:

$$
\tau=\frac{\phi}{\alpha(1-\phi) \rho_{\mathrm{s}}} .
$$

With $\phi=0.38, \alpha=0.02510^{-3} \mathrm{~m}^{3} / \mathrm{kg} \mathrm{h}$ and $\rho_{\mathrm{s}}=1886 \mathrm{~kg} / \mathrm{m}^{3}$ a typical filling time $\tau$ of $13 \mathrm{~h}$ is obtained.

In Eq. (17) the time elapsed since the start of the test appears, whereas in Eq. (55) the elapsed time minus the time needed to saturate the column is figuring. This time is easy to measure as it is the time measured from the instant the first leachant leaves the column. From Table 2 one can see that the times corresponding to the filling of flasks 5 , 6 and 7 is much larger than $\tau$, which amounts to $13 \mathrm{~h}$ only. So, for large $t_{j}$ and $t_{n}$, $\sqrt{ }\left(t_{n}-\tau\right)$ can be approximated by $\sqrt{ } t_{n}$. This approximation also holds for all $q$, as both $t_{j}$ and $\tau$ are inversely proportional to $q$ (and $\alpha$ ). The time $t_{n}$ is the time measured from the start of the test and is also easy to measure.

Next, the contaminant mass fraction in the material and the mass flux are specified. The first order approximation of the contaminant mass fraction in the column follows from Eqs. (23), (24), (31) and (47) as:

$$
C_{\mathrm{s}}(t \geq 0,0 \leq x \leq \chi(t))=C_{\mathrm{s} 0}-2 C_{\mathrm{s} 0} \frac{a}{1-\phi}\left(\frac{\mathbb{D}_{\mathrm{s}}}{\pi}\right)^{1 / 2} \sqrt{ }(t-\Upsilon(x)) .
$$

The first order approximation of the mass flux follows from Eqs. (2), (28), (48) and (59):

$$
\dot{m}(t \geq 0,0 \leq x \leq \chi(t))=\rho_{\mathrm{s}} C_{\mathrm{s} 0}\left(\frac{\mathbb{D}_{\mathrm{s}}}{\pi(t-\Upsilon(x))}\right)^{1 / 2} .
$$

Eq. (60) reveals that the mass flux depends on both the time and the position in the column.

In the literature, e.g. [4], also the measured ratio of leached contaminant and material mass, $U=M_{1}\left(t_{n}\right) / m_{0}$, is related to the so called $L / S$ ratio. This is the quotient of totally collected leachant and material mass, i.e. $\sum L_{j} / m_{0}$. Employing Eqs. (55) and (57) and:

$$
L / S=\sum_{j=1}^{n} L_{j} / m_{0}=\frac{q\left(t_{n}-\tau\right)}{m_{0}},
$$

it follows that

$$
U(L / S)=\frac{2 C_{\mathrm{s} 0} a}{(1-\phi) \sqrt{ } \alpha}\left(\frac{\mathbb{D}_{\mathrm{s}}}{\pi}\right)^{1 / 2} \sqrt{ }(L / S) .
$$

Eq. (62) reveals that lower $U$ as a function of $L / S$ are obtained if $a$ is reduced and the flushing velocity, governed by $\alpha$, is enhanced.

[15] derived empirically an equation for the leaching of granular materials which was based on the leaching of monoliths, reading:

$$
M_{1}\left(t_{n}\right)=2 \rho_{\mathrm{s}} C_{\mathrm{s} 0} a l \frac{\pi}{4} d_{\mathrm{i}}^{2}\left(\frac{\mathbb{D}_{\mathrm{s}}}{\pi}\right)^{1 / 2} \sqrt{ } t_{n}
$$


In their paper al $(\pi / 4) d_{\mathrm{i}}^{2}$ was replaced by equivalent $A$, i.e. the total exposed surface, and $\rho_{\mathrm{s}} C_{\mathrm{s} 0}$ by equivalent $M_{\mathrm{s} 0} / V$, where $M_{\mathrm{s} 0}$ is the contaminated mass initially present in the packed bed and $V$ the total volume of all grains. Comparing Eq. (63) with Eq. (55) one can see that in the former equation $\sqrt{ } t_{n}$ appears, whereas it should read $\sqrt{ }\left(t_{n}-\tau\right)$.

Experiments were also reported by Brown et al. ([15]) using pulverized mortar containing contaminants, which were leached in a column and via a serial batch leaching test. From both experiments diffusion coefficients were determined with Eq. (17) for the batch leaching test and with Eq. (63) for the column test. Their computed diffusion coefficients of the column test, however, should be corrected with a factor $t_{n} /\left(t_{n}-\tau\right)$. Accordingly, $\tau$ has to be determined. It follows from $q=0.2 \mathrm{~mL} / \mathrm{min}, d_{\mathrm{i}}=47 \mathrm{~mm}$, $\phi=0.4, l=57 \mathrm{~mm}$ and Eqs. (25) and (52) that $\tau=$ nearly $1 / 3$ day. For the computed $\mathbb{D}_{s}$ this implies that it it would be a factor $3 / 2$ larger for the first day of extraction $\left(t_{n}=1\right.$ day) and a factor $6 / 5$ for the two day extraction if the correction $t_{n} /\left(t_{n}-\tau\right)$ is invoked.. These corrections imply that the $L X\left({ }^{10} \log \left(\mathrm{cm}^{2} / \mathbb{D}_{\mathrm{s}}\right)\right.$ becomes 0.18 and 0.08 smaller for the one day and two day extraction test, respectively. For the 10 day leaching test the effect on $L X$ is -0.015 . These subtractions help to make the unclarified difference in measured $L X$ by batch and column tests, appearing in Table 2 of [15], a little smaller.

Finally, the magnitude of $\epsilon_{1}$ and $\epsilon_{2}$ should be assessed and verified whether these numbers are small indeed. To this end, Eqs. (36) and (37) are rewritten as:

$$
\begin{aligned}
& \epsilon_{1}=\frac{\rho_{\mathrm{s}} K_{\mathrm{p}} s}{2 \sqrt{\pi}} \frac{1-\phi}{\phi}\left(\frac{4 \mathbb{D}_{\mathrm{s}} t}{d_{\mathrm{p}}^{2}}\right)^{1 / 2}\left(\frac{\tau}{t}\right)^{1 / 2}, \\
& \epsilon_{2}=\frac{s}{2 \sqrt{\pi}}\left(\frac{4 \mathbb{D}_{\mathrm{s}} t}{d_{\mathrm{p}}^{2}}\right)^{1 / 2}\left(\frac{\tau}{t}\right)^{1 / 2},
\end{aligned}
$$

where Eqs. (20) and (25) have been substituted. The factors $4 \mathbb{D}_{\mathrm{s}} t / d_{\mathrm{p}}^{2}$ and $\tau / t$ are much smaller than unity, and $(1-\phi) / \phi$ and $s / 2 \sqrt{ } \pi$ are of order unity. Hence, $\epsilon_{2}$ is much smaller than unity and it is very likely that also $\epsilon_{1}$ is small since it equals $\epsilon_{2} \rho_{\mathrm{s}} K_{\mathrm{p}}(1-$ $\phi) / \phi$.

\section{Model generalization}

In Sections 2 and 3 mass transfer has been assumed to be proportional to $1 / \sqrt{ } t$. In practice other mass transfer kinetics are also encountered, e.g. see [16]. In general, this mass transfer coefficient can be expressed as:

$$
k_{\mathrm{s}}=\lambda t^{\mu-1} \text {. }
$$

Applying this mass transfer coefficient in the analysis of Section 2 yields as total mass of leached contaminant after $n$ leaching cycles:

$$
M_{1}\left(t_{n}\right)=\frac{\rho_{\mathrm{s}} A C_{\mathrm{s} 0} \lambda}{\mu} t_{n}^{\mu},
$$


and the analysis of Section 3, with Eqs. (66) and (62) results in:

$$
M_{1}\left(t_{n}\right)=\frac{\rho_{\mathrm{s}} C_{\mathrm{s} 0}}{\mu} a l \frac{\pi}{4} d_{\mathrm{i}}^{2} \lambda\left(t_{n}-\tau\right)^{\mu}
$$

and

$$
U(L / S)=\frac{C_{\mathrm{s} 0} a}{\mu(1-\phi) \sqrt{ } \alpha} \lambda(L / S)^{\mu},
$$

respectively. One can readily verify that for $\lambda=\sqrt{ }\left(\mathbb{D}_{\mathrm{s}} / \pi\right)$ and $\mu=1 / 2$ Eqs. (67)-(69) reduce to Eqs. (17), (55) and (62), respectively. Furthermore, one can see that for the mass transfer coefficient used here, again $t_{n}-\tau$ appears in the equation for the column test and $t_{n}$ in the monolith test.

When $\log U$ is set out graphically against $\log (L / S)$, a linear relationship is obtained whereby $\mu$ represents the slope of the line. During column leaching experiments of several fly ashes for most of their contaminants [4] indeed measured linear relationships. With the help of Eq. (69) it would be possible to assess both $\mu$ and $\lambda$.

\section{Conclusions}

The semiinfinite diffusion model is employed to describe the leaching of contaminants from monolithic and granular materials, which is allowed for small $4 \mathbb{D}_{\mathrm{s}} t / b^{2}$ and $4 \mathbb{D}_{\mathrm{s}} t / d_{\mathrm{p}}^{2}$, respectively. The considered processes are encountered in leaching tests such as prescribed by the Dutch standards NEN 7343 ([2]) and NEN 7345 ([1]). The leaching models issue from unsteady contaminant mass transfer between solid material and leachant, which are in nonequilibrium, and account for the accumulation of the contaminant in the leachant. For the monolithic material this approach results in analytical expressions for the contaminant concentration in leachant and material versus time. For small $4 \mathbb{D}_{\mathrm{s}} t / b^{2}\left(1+S / K_{\mathrm{p}} L\right)$, the solution can be simplified, yielding the original expressions of [5].

The leaching model of the granular material in the packed column results in a moving boundary problem. A suitable transformation yields a set of partial differential equations. Employing an asymptotic technique, based on small $4 \mathbb{D}_{\mathrm{s}} t / d_{\mathrm{p}}^{2}$, approximate analytical expressions for the contaminant concentration in leachant and material versus time and position in the column are obtained.

The resulting equations are used to determine the mean mixed concentration in the flasks and the total mass of leached contaminant versus time. Likewise for the monolith, the total mass of leached contaminant from the granular material is parabolic in time. The present model enables the computation of an effective diffusion coefficient from the leaching test, which in turn can be used to assess the long-term leaching behavior of granular materials containing hazardous contaminants. It is demonstrated that by choosing an optimal grain shape and size, as well as column size (possible within the constraints imposed by the Dutch standard) favorable leaching results can be obtained.

The obtained expressions are suited to assess the effectiveness of immobilization of contaminated grains (e.g. a fly ash) immobilized in a monolithic material (e.g. concrete). 
By comparing the effective diffusion coefficient of the untreated grains and from the monolith, and accounting for the dilution effect, it is possible to quantify the resistance of the material against leaching and hence, its binding capacity of the contaminants.

Though attention is restricted here to modeling of the Dutch standards, the results of the present analysis can easily be applied to similar tests which are into use in other countries. Furthermore, in many leaching experiments it is observed that the amount of leached contaminant does not exactly follow the square root of time. Accordingly, the models are generalized to situations where the mass flux is proportional to any power function of $t$. This extension of the model also yields compact and practical equations which relate the amount of leached contaminant and the test conditions.

\section{Notation}

$\begin{array}{lll}A & \text { interfacial area between material and surrounding leachant } & {\left[\mathrm{m}^{2}\right]} \\ a & \text { specific interfacial area in column } & {\left[\mathrm{m}^{-1}\right]} \\ b & \text { characteristic length of monolith } & {[\mathrm{m}]} \\ C_{\mathrm{s}} & \text { mass of contaminant per dry mass of material } & \\ c_{\mathrm{l}} & \text { contaminant concentration in leachant } & {\left[\mathrm{kg} \mathrm{m}^{-3}\right]} \\ c_{\mathrm{s}} & \text { contaminant concentration, Eq. }(29) & {\left[\mathrm{kg} \mathrm{m}^{-3}\right]} \\ \mathbb{D}_{\mathrm{s}} & \text { diffusion coefficient of contaminant in material } & {\left[\mathrm{m}^{2} \mathrm{~s}^{-1}\right]} \\ d_{\mathrm{i}} & \text { inner diameter of column } & {[\mathrm{m}]} \\ d_{\mathrm{p}} & \text { mean grain size of granular material } & {[\mathrm{m}]} \\ g & \text { acceleration due to gravity } & {\left[\mathrm{ms}^{-2}\right]} \\ i & \text { number of replenishments } & \\ j & \text { number of filled flasks } & \\ K_{\mathrm{p}} & \text { partition coefficient } & {\left[\mathrm{kg}^{-1} \mathrm{~m}^{3}\right]} \\ k_{\mathrm{s}} & \text { mass transfer coefficient in the material } & {\left[\mathrm{ms}^{-1}\right]} \\ L & \text { volume of leachant } & {\left[\mathrm{m}^{3}\right]} \\ L / S & \text { ratio of leachant volume and material mass, Eq. }(61) & {\left[\mathrm{kg}^{-1} \mathrm{~m}^{3}\right]} \\ M_{1} & \text { mass of leached contaminant } & {\left[\mathrm{kg}^{3}\right]} \\ M_{\mathrm{s}} & \text { mass of contaminant in material } & {\left[\mathrm{kg}^{-1}\right.} \\ m_{0} & \text { mass of granular material in column } & {\left[\mathrm{kg}^{-1}\right.} \\ \dot{m} & \text { mass flux } & {\left[\mathrm{kg} \mathrm{m}^{-2} \mathrm{~s}^{-1}\right]} \\ q & \text { leachant volume flow rate } & {\left[\mathrm{m}^{3} \mathrm{~s}^{-1}\right]} \\ S & \text { monolith mass } & {\left[\mathrm{kg}^{-1}\right.} \\ S & \text { shape factor of granular material } & \\ T & \text { transformed coordinate, Eq. }(31) & \\ t & \text { time } & {[\mathrm{s}]} \\ U & \text { ratio of leached contaminant mass and material mass } & \\ u_{1} & \text { superficial leachant velocity } & {\left[\mathrm{ms}^{-1}\right]} \\ V & \text { volume of material } & {\left[\mathrm{m}^{3}\right]} \\ w_{\mathrm{i}} & \text { mass fraction of grains on a sieve } & \\ X & \text { transformed coordinate, Eq. }(30) & \\ & & \end{array}$


$\begin{array}{lll}x & \text { coordinate } & {[\mathrm{m}]} \\ z_{\mathrm{i}} & \text { sieve diameter } & {[\mathrm{m}]}\end{array}$

\subsection{Greek symbols}

$\epsilon \quad$ perturbation quantity, Eqs. (10) and (36) or Eq. (37)

$\lambda$ mass transfer factor

$\mu \quad$ mass transfer power coefficient

$\rho_{\text {s }}$ material density

$\tau$ time needed to saturate the column, Eq. (25)

$\phi$ porosity

$\chi$ front position of leachant saturated part of the column, [m] Eq. (23)

$T \quad$ time needed for the leachant front to reach a given 29 position, Eq. (24)

$\left[\mathrm{ms}^{-\mu}\right]$

$\left[\mathrm{kg} \mathrm{m}^{-3}\right]$

[s]

$[\mathrm{m}]$

[s]

\subsection{Subscript}

0 initial condition

\subsection{Superscript}

- mean

\section{References}

[1] NEN 7345, Leaching characteristics of solid earthy and stony building and waste materials, Determination of the leaching of inorganic components from buildings and monolithic waste materials with the diffusion test, Nederlands Normalisatie Instituut, Delft (in Dutch) (1995).

[2] NEN 7343, Leaching characteristics of solid earthy and stony building and waste materials, Determination of the leaching of inorganic components from granular materials with the column test. Nederlands Normalisatie Instituut, Delft (in Dutch) (1995).

[3] ANSI/ANS 16.1, Measurement of the leachability of solidified low-level radioactive wastes by a short term test procedure, American Nuclear Society, La Grange Park (1986).

[4] H.A. Van Der Sloot, Waste Management, 16 (1996) 65.

[5] H.W. Godbee and D.S. Joy, Assessment of the Loss of Radioactive Isotopes from Waste Solids to the Environment, Part 1, Background and Theory, Oak Ridge National Laboratory ORNL/TM-4333, Oak Ridge, TN (1974).

[6] K.B. Harvey, C.D. Litke and C.A. Boase, Nuclear Waste Management, Proc. 2nd Int. Symp. in Chicago, 1983, Advances in Ceramics, Vol. 8, 1984, p. 496.

[7] M.W. Grant and G.B. Merrell, Conference Waste Management '85: Waste Isolation in the U.S., Technical Programs and Public Education, Tucson, 1985, Vol. 3, University of Arizona, AZ, 1985, p. 189.

[8] M. Hinsenveld, A shrinking core model as a fundamental representation of leaching mechanisms in cement stabilized waste, Ph.D. Thesis, University of Cincinnati (1992).

[9] J. Crank, The mathematics of diffusion, Oxford Univ. Press, Oxford, 1956.

[10] R.B. Bird, W.E. Stewart and E.N. Lightfoot, Transport Phenomena, Wiley, New York, 1960. 
[11] G.M. Fair and L.P. Hatch, J. Am. Water Works, 25 (1933) 1551.

[12] B.S. Baclic, and P.J. Heggs, Int. J. Heat Mass Transfer, 28 (1985) 1965.

[13] W. Nusselt, Z. Ver. dt. Ing., 55 (1911) 2021 (in German).

[14] M. Van Dyke, Perturbation methods in fluid mechanics, Parabolic Press, Stanford, 1975.

[15] T.M. Brown, P.L. Bishop and D.L. Gress, in D. Lorenzen, R.A. Conway, L.P. Jackson, A. Hamza, C.L. Perket and W.J. Lacy (Eds.), Proc. Hazardous and Industrial Solid Waste Testing and Disposal, Vol. 6, ASTM STP 933, American Society for Testing of Materials, Philadelphia, 1986, p. 79.

[16] F. Medici, C. Merli, G. Scoccia and R. Volpe, in T.M. Gilliam and C.C. Wiles (Eds.), Proc. Stabilization and Solidification of Hazardous Radioactive and Mixed Wastes, Vol. 2, ASTM STP 1123, Philadelphia, 1992, p. 171 . 